\title{
A PROPOSITO DE LAS COMUNIDADES ACADEMICAS
}

Dora Cardona Rivas

Profesora Facultad de Odontología. U. A. M.

1 CONOCIMIENTO, ha jugado papel central en procesos de desarrollo acelerado de otros países. Las innovaciones de los países desarrollados muestran una tendencia creciente a suprimir las ventajas comparativas que poseen los países latinoamericanos en la producción de bienes básicos de origen natural, situación a la que fueron relegados los países del tercer mundo. Desde antes del siglo pasado, la especialización de estos países en la producción de materia prima, impidió que se gestaran políticas, programas y proyectos que permitieran el desarrollo económico, político y social requerido para solucionar sus propios problemas. Se creó así una situación de dependencia que nos ha mantenido en las condiciones de países del tercer mundo, pobres y subdesarrollados.

Esta situación se ha reflejado igualmente a nivel de la cultura, lo cual se ha expresado en políticas educativas que refuerzan el carácter de dependencia observado a nivel económico, en la medida en que la ausencia de creación y producción de conocimiento predomina en 
todos los niveles educativos. Clara expresión de lo anterior es la escasa importancia que la universidad colombiana ha dado al desarrollo de las aptitudes y actitudes requeridas para la investigación a nivel de pregrado y a la formación de investigadores, en los cuales se promueva el conocimiento como una de las formas de superar el subdesarrollo. La dependencia tecnológica del país, centrada en la transferencia, ha relegado la contribución de la Universidad al desarrollo de la ciencia y la tecnología, habiéndose convertido la ciencia en nuestro medio en una adaptación y consumo de tecnologías, sin tener en cuenta las posibilidades del país para acceder al desarrollo científico, tecnológico y social re-
-...En este contexto, se plantea como la Universidad debe desempeñar un papel preponderante en cuanto debe $\ll$ ser la orientadora de la sociedad y comprometida con las realidades nacionales... 9 bia en el nuevo escenario mundial de internacionalización del capital, del trabajo y del mercado que se encuentra en gestación.

Tal como lo señala Colciencias, en este proceso de modernización, debe lograrse la articulación entre quehacer productivo, científico y tecnológico y el impacto social. «La ciencia debe incorporarse a la práctica cotidiana de la sociedad y contribuir a mejorar la calidad de vida de la población». (Colciencias).

En este contexto, se plantea como la Universidad debe desempeñar un papel preponderante en cuanto debe «ser la orientadora de la sociedad y comprometida con las querido para solucionar las realidades nacionales; ser conciennecesidades propias.

El desfase entre el contexto mundial y el contexto latinoamericano y el colombiano en particular, en todos los campos de la actividad humana, ha impulsado un proceso de modernización acelerada y de apertura, cuyo propósito es buscar una inserción favorable de Colomcia crítica y constructivista de una sociedad sujeta a grandes desequilibrios y cambios acelerados, defensora de libertades y derechos en la búsqueda de liberación de los pueblos; pasaporte para el ingreso efectivo a una vida cada vez más internacional y urgida de expresiones colectivas y de solidaridad supranacionales» (López G., 1991). 
Si bien el planteamiento anterior no es ajeno a la Universidad, han sido escasos los aportes que ésta en general ha dado al país, a excepción de algunas que han logrado acreditar una mayor tradición investigativa y académica.

En procura de hacer de las Universidades verdaderos centros de desarrollo, la Ley 30 de Educación Superior y la Política de Ciencia y Tecnología, señalan el marco de referencia desde el cual, las instituciones de Educación Superior deben proyectarse.

Con relación a la ley 30 de 1992 , son universidades las instituciones que acrediten su desempeño con criterio de universalidad, en las siguientes actividades: la investiga- ción científica o tecnológica; la formación académica en profesiones o disciplinas; la producción, desarrollo y transmisión del conocimiento y de la cultural universal y regional.

De acuerdo a lo anterior, en varios capítulos y numerales de la ley, se destacan aspectos prioritarios como la prelación de la formación integral de los colombianos; la creación, desarrollo y gestación del desarrollo científico, cultural, económico, político y ético a nivel nacional y regional; la promoción de la unidad nacional, la descentralización, la integración regional, y la cooperación interinstitucional con miras a que las diversas zonas del país dispongan de los recursos humanos y de las tecnologías apropiadas que
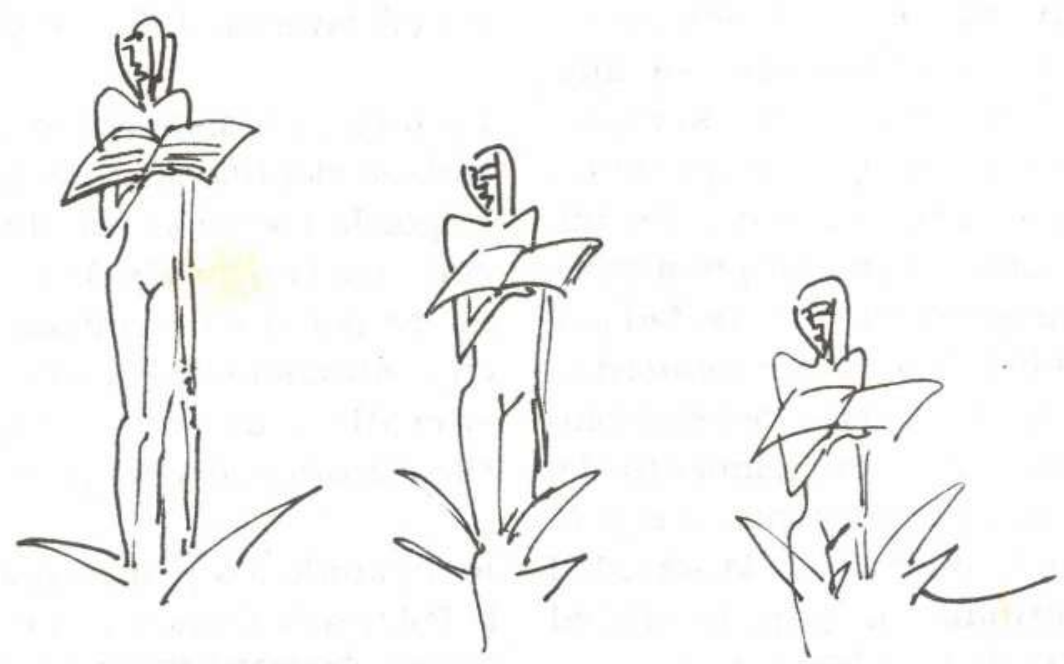
les permitan atender adecuadamente sus necesidades.

Los objetivos de la Ley 30 , concuerdan con la política de Ciencia y Tecnología propuesta por Colciencias, la cual contempla las siguientes líneas estratégicas:

1. Impulsar la innovación tecnológica.

2. Consolidar la capacidad científica nacional.

3. Reformar el sistema de ciencia y tecnología, en donde cobra gran importancia la adecuación del sistema universitario colombiano. Dicha adecuación ha de contemplar al menos los siguientes aspectos: a) la formación de profesionales capaces de participar en el desarrollo del conocimiento, y b) vincular su producción científica a programas estratégicos fundamentales requeridos por el país, en cuanto se propone integrar no sólo los sectores gubernamentales respectivos, sino también, a estos con las universidades y el sector productivo. Tríada gobierno, universidad (comunidad cientítica) e industria.

4. Cambiar la relación de los colombianos con el conocimiento. La ciencia debe incorporarse a la práctica cotidiana de la sociedad y contribuir a mejorar la calidad de vida de la población.
Dentro del contexto que plantea la política de ciencia y tecnología, se busca incorporar el desarrollo de la ciencia y la tecnología como elemento dinamizador de los procesos de desarrollo socioeconómico. «Dentro del esfuerzo para modernizar la nación, para mejorar la competitividad de nuestro aparato productivo, es necesario preocuparse por tener una base tecnológica muy fuerte que lo respalde; rehuyendo la tendencia que con facilidad se impone, de presumir que la adecuación tecnológica equivale a la adquisición de bienes de capital de productos de tecnología extranjera. Se requiere para la transferencia, la implantación, asimilación, desagregación y creación de tecnologías, una base sólida de conocimientos fundamentada en las disciplinas científicas sobre las que está construído el saber tecnológico» (Villaveces, J. L., 1992).

La tarea consiste en impulsar el trabajo científico, el reconocimiento por la sociedad, su vinculación con el sector productivo, su valoración por el sector público y lograr en consecuencia avances en el desarrollo de la capacidad científico-técnica del país.

Retomando los planteamientos de la Política de Ciencia con relación al punto, CONSOLIDAR LA CAPACI- 

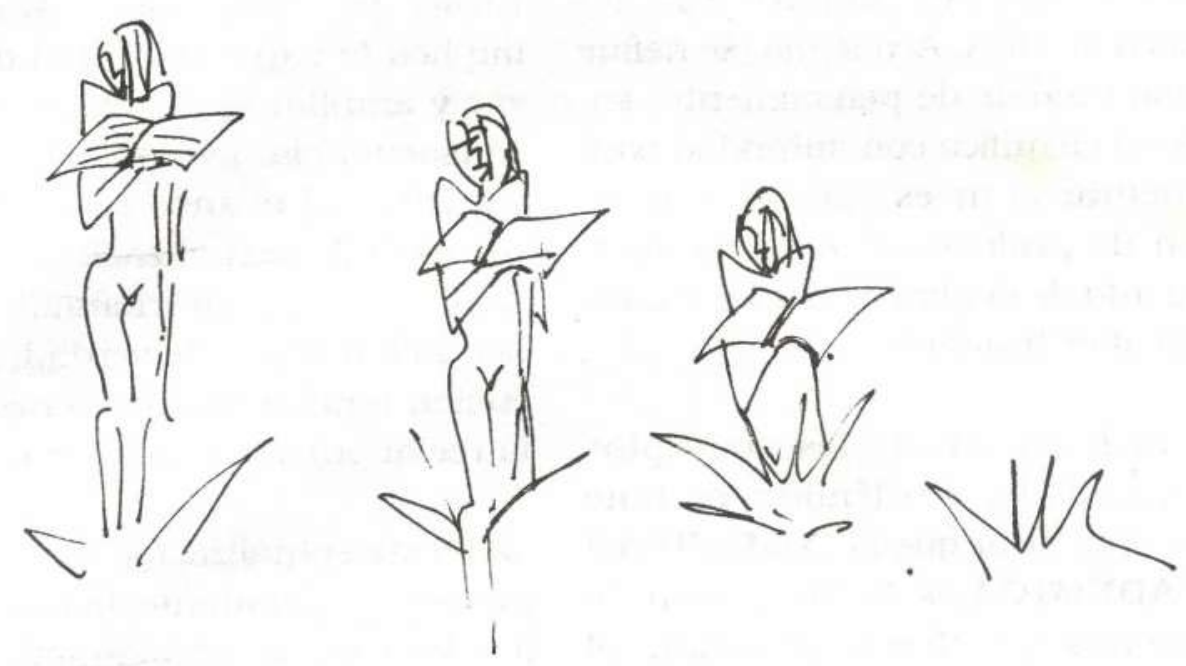

DAD CIENTIFICA NACIONAL, Se realizarán acciones orientadas a incentivar a los investigadores, fortalecer las instituciones de investigación, atender a la formación de redes de investigadores (a nivel regional nacional e internacional) y a la construcción de comunidades de investigadores (Colciencias).

Las acciones planteadas en la política de Ciencia y Tecnología, son un reto para las Universidades con poca tradición académica e investigativa y requieren que toda la comunidad universitaria encamine sus esfuerzos a propiciar el desarrollo de la ciencia, la técnica, el arte, las humanidades, en los campos propios de sus objetos de conocimiento y formación.
Como estrategia se pretende la multiplicación y fortalecimiento de grupos interesados y comprometidos con el avance humanístico-científico-tecnológico, existentes en la Universidad y propiciar la consolidación de auténticas COMUNIDADES ACADEMICAS $Y$ CIENTIFICAS.

Pero, QUE ES UNA COMUNIDAD ACADEMICA?

Si nos basamos en la etimología de las palabras, el término comunidad (del latín communitis, - tatis) significa entre otras: calidad de común, propio de todos, junta o congregación de personas que viven unidas, asociación de personas unidas por...

Académico (del latín academicus) significa, lo perteneciente o relativo 
a las academias; propio o característico de ellas. Academia, se define como escuela de pensamiento; sociedad científica con autoridad para fomentar la investigación, asociación de profesionales dedicada al fomento de cualquiera de las ramas del saber humáno.

La articulación de los conceptos comunidad y académica, permite conceptualizar que la COMUNIDAD ACADEMICA es la asociación de personas que tienen en común el interés y las potencialidades para incursionar en un determinado campo del saber o un sector de la realidad, de una manera que la hace portadora de la tradición, las formas de investigación, de trabajo y de valores sobre el área, disciplina, problema o realidad alrededor del cual se ha constituido.

\section{- Las acciones plan-} teadas en la política de Ciencia y Tecnología, son un reto para las Universidades con poca tradición académica e investigativa ...?
El ser portadora de la tradición implica la capacidad para conservar y ampliar el conocimiento; en consecuencia, avanza en el saber vigente. Tal avance se produce a través de la actualización, la posición crítica, el cuestionamiento de las pretensiones de verdad, la discusión fundamentada en razones y la reelaboración conceptual.

La reconceptualización debe ser un proceso permanente hecho sobre los desarrollos teórico-metodológicos en el contexto mundial, nacional y regional, sin olvidar el papel del conocimiento en el desarrollo de la sociedad. Elemento importante en este proceso, es acceder a las formas de comunicación y difusión del conocimiento.

La Ciencia es una empresa colectiva, y como tal requiere de la comunicación permanente, de la confrontación de ideas, de la relación con otras comunidades y con la sociedad en general. «El investigador tiene que trabajar en relación con la información, con los pares, con las posibilidades de crítica mutua, de aceptación de la crítica» (Villaveces, J. L. 1990) y toma de postura frente a ella.

Se trata de establecer una relación dialéctica con otras teorías, métodos. de trabajo, valoraciones, 
posibilidades de aplicación, en una forma tal que se enriquezca el conocimiento de los objetos propios de la comunidad académica.

Es así como la comunidad académica puede llegar a ser Escuela de Pensamiento e Investigación en la medida en que contribuye a la acumulación, sistematización. reelaboración, difusión y confrontación del conocimiento.

En el momento actual, en que se reconoce en todos los ámbitos de la sociedad, que no hay un verdadero desarrollo científico en el país, es imperativo operacionalizar el nuevo concepto de comunidad académica. Las razones para hacerlo se fundamentan en el hecho de que la Ciencia no es una empresa individual y ello lo demuestran entre otros los siguientes aspectos:

- La complejidad de la realidad en todos los ámbitos de la vida y el desarrollo humano.

- La expansión y rapidez de la producción científica en el mundo. Expertos señalan que la información se duplica aproximadamente cada siete años.

- La primacía de la competitividad en todos los campos del desarrollo y el hecho de que tal competitividad

\section{La Ciencia es}

una empresa colecti-

va, y como tal

requiere de la comu-

nicación

permanente ... está vinculada al desarrollo de conocimiento y la capacidad de integración, diferenciación y aplicación del conocimiento construido. Esta primacía de la competitividad no se justifica exclusivamente a la utilización del conocimiento como fuerza productiva, sino que está intimamente ligada a posibilitar y lograr que los individuos y los grupos sociales accedan a una mejor calidad de vida. Es por ello que se reconoce la necesidad de la apropiación crítica del conocimiento y su validación.

- La capacidad de la mayoría de las instituciones educativas y del país. de consolidar los diversos componentes de la infraestructura requeridas para el desarrollo cien- 
tífico, en forma individual.

- La necesidad de aprehender el conocimiento requerido para la solución de los problemas específicos, para entender los fundamentos de las tecnologías y para poder estar en el punto de desarrollo de nuevas teorías.

Lo anterior hace imprescindible el dominio de los conocimientos propios de un área del saber, dominio que necesariamente se ubica en las fronteras, en los límites, en lo inseguro, en lo que no se conoce. En fin, en el espacio en el que surgen los interrogantes.

- Además el aspecto central que es preciso destacar con relación a la capacidad científica, es que debe contribuir al desarrollo cultural, al de las instituciones sociales y políticas; propiciar la formación de individuos libres y de mayor autonomía que desplieguen las facultades humanas (Colciencias). Es así, como el conocimiento debe ser abordado desde las posibilidades mismas de transformación de contextos particulares de acción. La actividad científica tiene una función social. Tal función ha de ser objeto obligado de discusión en las comunidades académicas.

Teniendo en mente la innegable justificación de las comunidades académicas, es pertinente señalar, que estas tienen un propósito fundamental que las identifica: LA CONSTRUCCION COLECTIVA DEL CONOCIMIENTO y esta construcción es una práctica social. Parte de la experiencia social y revierte sobre ella, transformándola.

El propósito de construir conocimiento (hacer ciencia), requiere entre otros de las siguientes condiciones:

- de la apropiación reflexiva y crítica del acervo bibliográfico o tradición de las ciencias, disciplinas o profesiones;

- del desarrollo de la capacidad para reconocer la transitoriedad de los sistemas de conceptos vigentes en un momento histórico particular, y orientarse a la búsqueda de sistemas cada vez más estructurados, más coherentes y más consistentes.

- de reconocer las diferentes formas para construir conocimiento, de acuerdo a la especificidad de los problemas por resolver;

- del hacer conciencia sobre la finalidad y el sentido de la ciencia; en otras palabras, se ocupa del como responder al compromiso con el desarrollo humano en todas sus dimensiones. 


\section{- Una comunidad académica, se inserta en la}

tradición escrita propia, accede a la información que se produce constantemente; al fundamento mismo de las formas de construcción de tal conocimiento; a las formas de trabajo y argumentación propias

Pero DE QUE SE OCUPA O CUAL ES EL OBJETO DE UNA COMUNIDAD ACADEMICA?

Una comunidad académica, en primer lugar define su objeto de estudio. Identificado su objeto, se inserta en la tradición escrita propia, accede a la información que se produce constantemente; al fundamento mismo de las formas de construcción de tal conocimiento; a las formas de trabajo y argumentación propias. Reconoce las limitaciones del saber monodisciplinarios para la explicación, comprensión o transformación de los problemas del medio. Se abre hacia otros saberes que enriquecen la comprensión y las posibilidades de transformación. Es decir evalúa, y decide sobre las posibildades de aplicación y la función social del conocimiento construido.
Es aquí, donde posiblemente cabría señalar que los objetos de estudio de las comunidades académicas, darán lugar a diferentes tipos de ellas:

- las que se ocupan de los saberes monodisciplinarios de las llamadas ciencias duras, que conducen a la superespecialización;

- y comundiades que se articulan sobre problemas de la praxis social, que ameritan el diálogo interdisciplinario, el acceso a otros saberes, y «donde se valida en sus consecuencias éticas el conocimiento construído» (Alvarado S. V. 1991).

Es pertinente señalar que al hablar de problemas de la praxis social, no se excluye el saber de las ciencias duras, sino que se articulan a los 
procesos de transformación social. Es el saber de las ciencias naturales, (ej: el saber biológico en la medicina) y de las matemáticas etc. en el contexto de una realidad social que puede ser transformada.

Como conclusión perentoria, podría decirse que la comunidad académica, evalúa, reconoce, reconstruye las realizaciones, competencias, límites e interrogantes que posibilitan su desarrollo (Mockus A., 1990).

Precisado el propósito y objeto de una comunidad académica, vale la pena preguntarse, QUE CONDICIONES SE REGUIEREN PARA LA CONFORMACION DE UNA COMUNIDAD ACADEMICA?

Si bien, como se mencionó antes, lo que permite la adhesión de los miembros de una comunidad académica se fundamenta en el interés y la calidad de común, este no es suficiente. Se precisa de la voluntad de construir colectivamente el conocimiento. Esta voluntad debe expresarse en la formación de espacios en los que se acceda a una cultura académica (forma de pensar, actuar e interrelacionarse), que:

- potencie la individualidad para acrecentar la producción colectiva; - priorice el desarrollo del talento y la productividad académica;
- propicie la cooperación y el respeto por la diferencia;

- priviligie la deliberación, la confrontación racional, argumentada desde las formas propias de construcción de los saberes en discusión; - revalúe permanentemente el conocimiento y construya nuevos objetos de conocimiento;

- fomente las formas de comunicación de la universidad como la tradición escrita;

- desarrolle la mentalidad para lo universal, lo internacional y los retos del desarrollo histórico;

- se genere compromiso, responsabilidad y reflexión.

Sobre la ORGANIZACION, CRECIMIENTO Y DESARROLLO DE LAS COMUNIDADES ACADEMICAS, pueden señalarse algunos aspectos.

La conformación de una comunidad académica se posibilita cuando se identifican interlocutores que comparten su interés y voluntad de trabajo en torno a objetos de estudios relativos a la ciencia, la técnica la cultura, etc. A este primer momento de identificación sigue la costrucción de los espacios de deliberación, crítica, legitimación y producción, como se mencionó previamente.

Tal como en todas las actividades 
que conforman la vida académica, es pertinente la planeación del proceso a seguir, definiendo: objetivos, metas, estrategias y precisar en el tiempo, los desarrollos que se pretenden lograr. Esta planeación es particularmente importante, para lograr la disciṕlina de trabajo necesaria para la producción académica, requisito sine qua non para la existencia de una comunidad académica.

Toda comunidad académica, debe estar dotada de su propia tradición escrita y su propio ámbito de discusión, expresado principalmente en sus publicaciones (textos, revistas, manuales). La tradición escrita, permite decantar los resultados siempre provisionales, siempre parciales, de las discusiones adelantadas hasta cierto momento. Permite no regresar permanentemente a 1 cuestionamiento del todo, crea una cierta posibilidad de acumulación de conocimiento.

Además, la tradición escrita potencia la discusión racional, ya que la escritura sustrae los conceptos o teorías de lo efímero del enunciado oral. La discusión en la academia es escrita. A través de la discusión racional se pretende llegar a un punto en el cual el curso de la discusión y su desenlace, pueden resultar relevantes y obligantes para quienes participan de ella. Mediante la combinación de la tradición escrita, la discusión racional y la reorientación de la acción derivada de esa discusión racional, una comunidad académica va ganando la competencia que se expresa en el logro de la AUTORIDAD, autoridad que se construye y reconoce por la validez de los propios méritos y que trasciende el ámbito de lo público a nivel regional, nacional o internacional.

La trascendencia de las comunidades académicas es el resultado de su capacidad para respaldarse en argumentos y evidencias que tendrán que ser reconocidas como válidas por cualquier interlocutor que se tome el trabajo de examinarlos.

- Toda comunidad académica, debe estar dotada de su propia tradición escrita y su propio ámbito de discusión ... 
A modo de conclusión y teniendo en cuenta el marco de referencia de atraso en el desarrollo científico, tecnológico y social del país, y la necesidad de abordar en forma inmediata la transformación de la actividad investigativa de la Universidad, con el fin de que pueda tener algún impacto al futuro, la conformación y consolidades de comunidades académicas en el ámbito universitario, es un paso ineludible.

El docente universitario debe ampliar su visión del mundo y de la sociedad actual, debe ubicar los avances de su profesión, disciplina o saber, redimensionar su praxis, confrontarse permanentemente con sus homólogos, compartir conocimientos, técnicas, productos, recursos, etc., en fin, asumir el compromiso de hacer de la construcicón de conocimiento el eje articulador de su práctica educativa, aportando al desarrollo que el país requiere.

\section{BIBLIOGRAFIA}

ALVARADO, S. V. La investigación social en los programas de Postgrado como estrategia de desarrollo social. El caso CINDE. Manizales: Nova University-Centro Internacional de Educación y Desarrollo Humano.
Tesis de grado presentada como requisito parcial para obtener el título de Doctor en Educación, 1992.

CADAVID, Gabriela. Apuntes tomados en el Seminario Taller de Gestión Unversitaria. Universidad de Antioquia-Universidad Autónoma de Manizales.

COLCIENCIAS-DPN. Ciencia y Tecnología para una sociedad abierta. 1990 .

LOPEZ G., citado por: ALVARADO, S. V. La investigación social en los programas de Postgrado como estrategia de desarrollo social. El caso CINDE. Manizales: Nova UniversityCentro Internacional de Educación y Desarrollo Humano. Tesis de grado presentada como requisito parcial para obtener el título de Doctor en Educación, 1992.

MOCKUS, Antanas. Presupuestos filosóficos y epistemológicos del privilegio del Currículo. Planteamientos y reflexiones alrededor del currículo en la Educación Superior, p. 133-186.

MOCKUS, Antanas. Fundamentos teóricos para una reforma de la Universidad. Educación y Cultura 21: 22-37, 1990. 